\title{
A Jaw-Dropping Diagnosis
}

Dina Griauzde, MD, MSc ${ }^{1,2 *}$, Rebecca Northway, MD²,3, Sarah Yentz, MD 1,2, Sanjay Saint, MD, MPH ${ }^{1,2}$, Nathan Houchens, MD ${ }^{1,2}$

This icon represents the patient's case. Each paragraph that follows represents the discussant's thoughts.

'Veterans Affairs Ann Arbor Healthcare System, Ann Arbor, Michigan; ²Department of Internal Medicine, University of Michigan Medical School, Ann Arbor, Michigan; ${ }^{3}$ Department of Pediatrics and Communicable Diseases, University of Michigan Medical School, Ann Arbor, Michigan.

A 73-year-old man presented to primary care for an annual examination. Four days prior, he noted right-sided sharp jaw pain such that he could not open his mouth nor chew solid food; it radiated from the right mandible to the ipsilateral temple. He also noted bilateral aching hip pain for several years that increased in severity in the prior 2 months. He reported an intentional weight loss of $9 \mathrm{~kg}$ over the past year, achieved through dietary modification. He denied fever, chills, and visual disturbance.

Acute onset of unilateral jaw pain that is worsened by chewing is a feature consistent with a temporomandibular disorder (TMD). TMD consists of musculoskeletal and neuromuscular conditions that affect the temporomandibular joints (TMJs), masticatory muscles, and associated tissues. Common symptoms of TMD include facial or ear pain, temporal headache, and TMJ dysfunction or discomfort. In addition to TMD, craniofacial pain has many possible etiologies such as dental pathology, neuralgias, sinus and otologic disorders, headache and migraine disorders, infections, rheumatologic conditions, and neoplasms.

Systemic etiologies for this patient's symptoms are a consideration given his age and concomitant worsening of chronic hip pain. Rheumatologic conditions such as giant cell arteritis (GCA) and polymyalgia rheumatica (PMR) are more common in adults older than 50 years of age and cause headache, jaw claudication, and pelvic girdle pain. Rarely, hematologic malignancies (eg, lymphoma), solid tumor metastases (eg, breast cancer, melanoma), and primary tumors of the head and neck (eg, nasopharyngeal carcinoma) can involve the mandible, TMJ, or parotid gland and result in symptoms of TMD.

Medical history was notable for hypertension and type 2 diabetes mellitus complicated by peripheral neuropathy. He smoked one pack of cigarettes daily for 40 years but quit 15 years prior. He drank 4 ounces of vodka each night.

On examination, temperature was $36.5^{\circ} \mathrm{C}$, heart rate 92 beats per minute, blood pressure $127 / 60 \mathrm{mmHg}$, respiratory

\footnotetext{
*Corresponding Author: Dina Griauzde, MD, MSc; Email: dhafez@med.umich. edu; Telephone: 734-845-5129; Twitter: @nate_houchens; @DinaGriauzde.

Published online first May 20, 2020.
}

Received: August 27, 2019; Revised: January 7, 2020;

Accepted: January 27, 2020.

(c) 2020 Society of Hospital Medicine DOI 10.12788/jhm.3389 rate 12 breaths per minute, oxygen saturation $98 \%$ on ambient air, and weight $118 \mathrm{~kg}$. Extraocular movements were intact, pupils were equal and reactive to light and accommodation, and there were no visual field deficits. Nondilated funduscopic examination revealed normal blood vessels, optic disc, and optic cup-to-disc ratio. Dentition was good with pink gingiva. Bilateral temples were nontender. There was normal range of motion and strength in the shoulders, hips, and lower extremities with no tenderness over the trochanters. Patellar and ankle reflexes were present and symmetric bilaterally. He had no rashes or ecchymoses.

The history of smoking, especially with concomitant alcohol intake, is a risk factor for head and neck cancer, and these malignancies can lead to facial pain. While the normal oral cavity exam argues against localized oral and dental causes of the patient's symptoms, direct fiberoptic endoscopy should be considered. The neck should be examined for lymphadenopathy. Normal vital signs point away from severe infection. The lack of findings in the head and musculoskeletal regions does not exclude systemic etiologies such as rheumatologic conditions or neoplasm. Complete blood cell count and markers of inflammation including erythrocyte sedimentation rate (ESR) and C-reactive protein (CRP) levels should be obtained. Hip and pelvic radiographs should be obtained to evaluate for hip osteoarthritis, fractures, or osseous lesions.

The appointment occurred during evening hours and the patient declined further evaluation until the following morning, at which time laboratory studies revealed normal serum levels of electrolytes, blood urea nitrogen, and creatinine. White blood cell (WBC) count was $6,800 / \mathrm{mm}^{3}$ with an immature granulocyte ratio of $1.8 \%$ (normal, $0.0-0.5 \%$ ), hemoglobin $13.2 \mathrm{~g} / \mathrm{dL}$, and platelet count 163,000/. $\mathrm{mm}^{3}$. ESR was $118 \mathrm{~mm} / \mathrm{hr}$ (normal, 0-15 mm/hr) and CRP was $1.5 \mathrm{mg} / \mathrm{dL}$ (normal, $0-0.75 \mathrm{mg} / \mathrm{dL}$ ). Radiographs of the hips and pelvis showed osteoarthritis of the bilateral hip joints and degenerative disc disease of the lower lumbar spine.

Granulocytosis may occur in response to infection, rheumatologic conditions, and hematologic malignancies such as chronic myelogenous leukemia. While infectious etiologies (eg, abscess, osteomyelitis) are the most common cause of an extremely elevated ESR level, this patient does not have other signs or symptoms of infection such as fever or leuko- 
cytosis. Therefore, other common causes for an extremely elevated ESR level should be considered, including malignancy (eg, multiple myeloma, lymphoma, metastatic solid tumor) and autoimmune conditions (eg, rheumatoid arthritis, vasculitis). While multiple myeloma is the most common malignant etiology for extremely elevated ESR, the patient lacks signs of this condition such as anemia, elevated creatinine, or osteolytic lesions on radiographic imaging. Osteoarthritis identified on the radiographs may contribute to the patient's hip pain but would not explain the patient's jaw pain, weight loss, granulocytosis, and elevated ESR. These findings, taken together with the patient's age, are most suggestive of GCA with possible coexisting PMR. Temporal artery biopsy should be obtained as it is the gold standard test for diagnosing GCA.

The patient was contacted by telephone that same day with laboratory test results. During the call, he endorsed increased jaw and temple pain. He was advised to proceed to the emergency department (ED) for timely evaluation and treatment.

Because GCA was being considered, ophthalmology performed an ocular examination in the ED, which demonstrated no signs of optic nerve or retinal ischemia. Computed tomography (CT) scan of the head and neck with intravenous contrast revealed no abscess or soft tissue abnormalities. Right temporal artery biopsy was performed.

The normal ocular examination does not exclude GCA, and temporal artery biopsy is appropriate. The mainstay of treatment for GCA is high-dose systemic glucocorticoids, which should not be withheld while awaiting biopsy results since ophthalmic artery inflammation may occur and threaten vision.

While GCA remains the leading diagnosis, malignant etiologies warrant further consideration because they are a common cause of extreme ESR elevation, particularly among older patients. The patient's cancer screening history should be reviewed. The normal CT scan of the head and neck reduces the likelihood of localized solid tumor etiologies; however, additional CT imaging of the chest, abdomen, and pelvis is warranted to evaluate for metastatic solid tumors or lymphoma.

A 10-day course of prednisone $60 \mathrm{mg}$ daily was prescribed for empiric treatment of GCA. The patient was discharged home with follow-up scheduled in rheumatology and primary care clinics. Pain in the jaw and temple resolved within several days.

Two weeks later, he presented to the rheumatology clinic. He noted 1 week of lower right back pain described as dull, aching, radiating to the lateral right hip, and occurring when transitioning from sitting to standing. He had no leg numbness, weakness, or change in bowel habits. Bladder habits were also unchanged, although he reported chronic urinary frequency and occasional incontinence. He reported further weight loss, this time an unintentional loss of $9 \mathrm{~kg}$. He noted frequent sweating but no fever.

He reported a normal colonoscopy within the prior 5 years.
Because these records were not available for review, a fecal immunochemical test was obtained and negative for hemoglobin. He had previously declined prostate cancer screening.

The resolution of jaw and temple pain with prednisone supports the presumed diagnosis of GCA. Up to half of patients with GCA may also have PMR, which can cause aching and stiffness in the arms, hips, and lumbar region, and pain may be abrupt in onset. However, PMR-related pain would be expected to improve rather than develop or worsen in the setting of high-dose glucocorticoid use. Therefore, other causes of acute-onset back pain must be considered.

While localized musculoskeletal etiologies such as lumbar muscle strain, radiculopathy, and vertebral compression fracture are possible, co-occurrence of unintentional weight loss and diaphoresis with elevated inflammatory markers suggests a systemic etiology. A neoplastic process with bony metastasis is possible. The reportedly normal colonoscopy and the negative fecal immunochemical test make colorectal cancer less likely. Inflammatory conditions such as ankylosing spondylitis and rheumatoid arthritis are also possible. Ankylosing spondylitis usually presents at a much younger age, however, and axial skeletal involvement in rheumatoid arthritis often involves the cervical spine and is usually seen after longstanding disease. Additionally, the hallmark of inflammatory back pain is morning stiffness which the patient does not endorse. Nonetheless, additional laboratory testing should include antinuclear antibody, rheumatoid factor, and anti-cyclic citrullinated peptide (anti-CCP) antibody. Vertebral osteomyelitis remains on the differential diagnosis, and repeat WBC count and inflammatory markers should be assessed. Lumbosacral radiographs should be obtained to rule out fracture.

Physical examination in the rheumatology clinic revealed a temperature of $37.0^{\circ} \mathrm{C}$, heart rate 100 beats per minute, blood pressure $146 / 72 \mathrm{mmHg}$, respiratory rate 12 breaths per minute, and oxygen saturation $98 \%$ on ambient air. Weight was $109 \mathrm{~kg}$. He was pale and diaphoretic. There was diffuse tenderness to palpation of the right-sided lumbar paraspinal muscles. Straight leg raise was negative bilaterally. Patellar reflexes and gait were normal.

Blood chemistries, renal function, and aminotransferase levels were normal. WBC count was $7,100 / \mathrm{mm}^{3}$, hemoglobin $8.0 \mathrm{~g} / \mathrm{dL}$, mean corpuscular volume $88.9 \mathrm{fL}$, platelet count $128,000 / \mathrm{mm}^{3}$, ESR $66 \mathrm{~mm} / \mathrm{hr}$, CRP $0.57 \mathrm{mg} / \mathrm{dL}$, alkaline phosphatase $438 \mathrm{IU} / \mathrm{L}$ (normal, 30-130 IU/L), and thyroid-stimulating hormone $0.925 \mathrm{mU} / \mathrm{L}$ (normal, 0.34-5.60 mU/L). Testing for antinuclear antibodies, rheumatoid factor, and anti-CCP antibody was unremarkable. Prostate-specific antigen (PSA) level was $2.2 \mathrm{ng} / \mathrm{mL}$ (normal, 0-4 ng/mL). Urinalysis was unremarkable. Antibodies to hepatitis $\mathrm{C}$ and Treponema pallidum were negative. Interferon gamma release assay was negative.

Findings of new onset anemia and thrombocytopenia, in combination with elevated ESR and alkaline phosphatase level, are concerning for disseminated intravascular coagulation (DIC) 

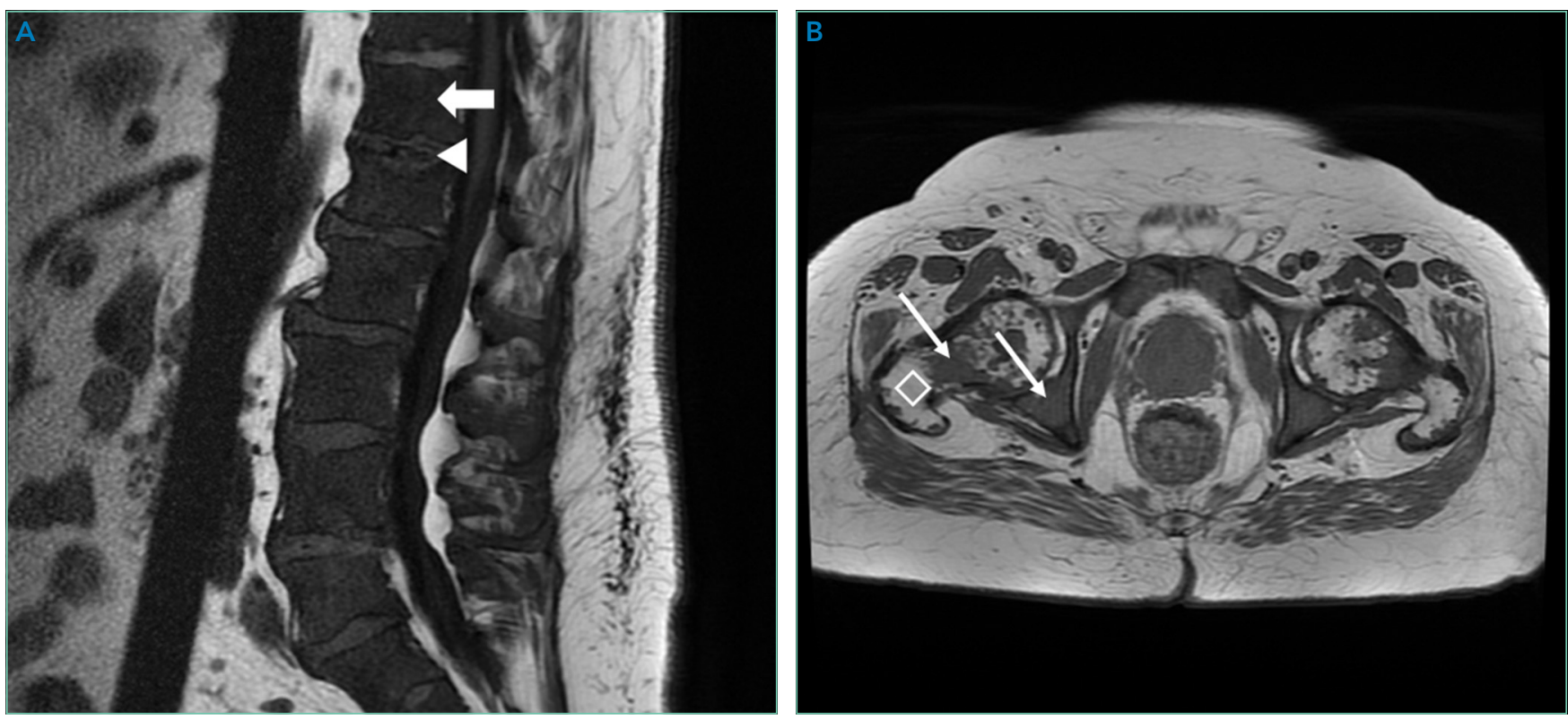

FIG 1. Magnetic Resonance Imaging (MRI) of the lumbar spine (left) and pelvis (right). A sagittal T1-weighted MRI of the lumbar spine shows bone marrow (thick arrow) that is darker than the adjacent intervertebral disc (arrowhead). An axial T1-weighted MRI through the pelvis shows normal fatty bone marrow signal in the right greater trochanter (diamond) and abnormal hypointense signal in the right femoral neck and acetabulum (thin arrows). These images demonstrate abnormal bone marrow replacement.

and microangiopathic hemolytic anemia (MAHA), bone marrow infiltration of a metastatic neoplasm, or ineffective hematopoiesis caused by myelodysplastic syndromes or myelofibrosis.

Laboratory evaluation should include iron studies, lactate dehydrogenase (LDH), haptoglobin, fibrinogen, D-dimer, reticulocyte count, and peripheral blood smear to assess for hemolysis and erythrocyte morphology. Advanced imaging with lumbosacral magnetic resonance imaging (MRI) should be obtained to evaluate for focal etiologies of back pain such as disc herniation, abscess, marrow infiltration, and infarction.

Additional laboratory studies revealed a gammaglutamyl transferase level of $49 \mathrm{IU} / \mathrm{L}$ (normal, 8-56 IU/L), LDH 288 IU/L (normal, 98-192 IU/L), haptoglobin 495 mg/dL (normal, $32-240 \mathrm{mg} / \mathrm{dL}$ ), fibrinogen $>700 \mathrm{mg} / \mathrm{dL}$ (normal, 225-550 mg/dL), D-dimer $693 \mathrm{ng} / \mathrm{mL}$ (normal, 200-250 ng/ $\mathrm{mL}$ ), serum iron $57 \mathrm{mcg} / \mathrm{dL}$ (normal, $33-150 \mathrm{mcg} / \mathrm{dL}$ ), total iron binding capacity $286 \mathrm{mcg} / \mathrm{dL}$ (normal, $250-450 \mathrm{mcg} / \mathrm{dL}$ ), ferritin 1,012 ng/mL (normal, 17.9-464 ng/mL), and reticulocyte count $2.9 \%$ (normal, $0.5-2.5 \%$ ). Coagulation studies and serum protein electrophoresis were normal. Erythropoietin level was $109 \mathrm{mIU} / \mathrm{mL}$ (normal, 4.0-20.0 $\mathrm{mlU} / \mathrm{mL}$ ). Peripheral blood smear demonstrated moderate anemia with $8 \%$ nucleated erythrocytes per white blood cell (normal, $0 \%$ ) and no circulating blasts.

MRI of the thoracolumbar spine and pelvis revealed diffusely abnormal bone marrow signal with multiple superimposed focal and poorly defined enhancing lesions along the lumbar spine marrow, sacrum, and bilateral iliac bones (Figure 1). Positron emission tomography/computed tomography (PET/ CT) scan showed no scintigraphic evidence of metabolically active neoplastic, paraneoplastic, or inflammatory disorder.
The elevated haptoglobin, normal coagulation studies, and absence of fragmented erythrocytes on peripheral smear exclude an intravascular hemolytic process. The patient's lower than expected reticulocyte count for the degree of anemia, elevated erythropoietin, and nucleated erythrocytes constitute a pattern that can be seen with bone marrow infiltration. There are no circulating blasts, making leukemia less likely. A solid organ tumor with bone metastases may cause enhancing lesions on MRI since this form of imaging is more sensitive than radiography for detecting skeletal malignancies. The negative $\mathrm{PET} / \mathrm{CT}$, however, does not reveal a primary tumor. Myelofibrosis is an infiltrative myeloproliferative disorder associated with nonspecific laboratory abnormalities, bone pain, weight loss, and night sweats that could cause diffuse MRI bone marrow signal alterations with normal PET/CT findings. However, myelofibrosis would not typically cause a significantly elevated ESR, and thus would be an unlikely cause for this patient's presentation.

Given the constellation of symptoms, hematologic abnormalities, and bone marrow infiltration on imaging, hematology should be consulted to perform a bone marrow biopsy to assist with definitive diagnosis

Bone marrow biopsy demonstrated metastatic adenocarcinoma consistent with prostatic origin (Figure 2). Bone scan demonstrated widespread osteoblastic metastases, which included the skull and temporal regions. These lesions were thought to be the cause of the patient's original presenting symptom of jaw pain.

The patient was started on androgen deprivation therapy, initially with degarelix and subsequently leuprolide shots and abiraterone with prednisone. PSA was $0.08 \mathrm{ng} / \mathrm{mL}$ after 3 

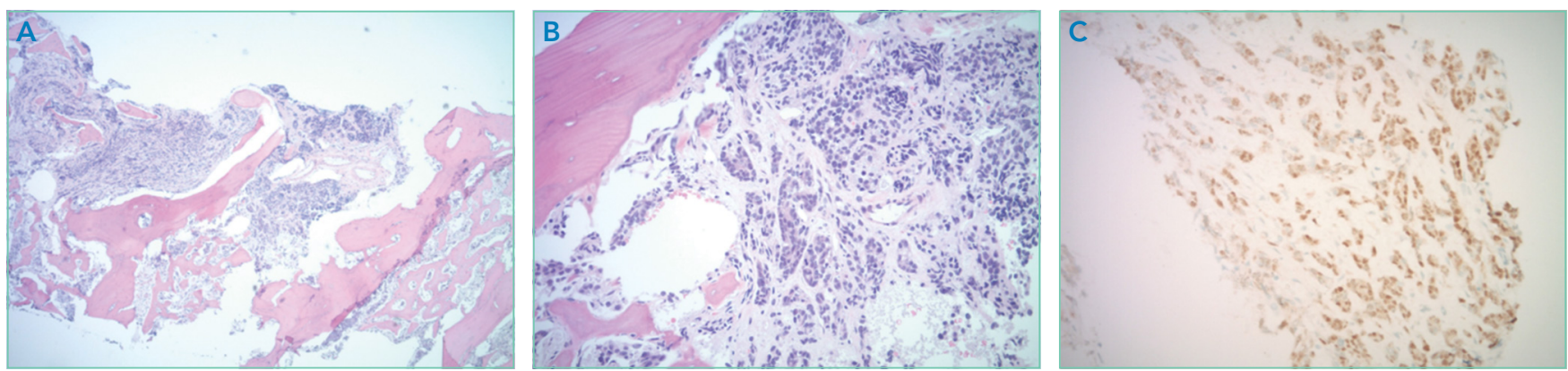

FIG 2. Bone marrow biopsy specimen. In Panel $A$, and in Panel $B$ at higher magnification, hematoxylin and eosin staining of a biopsy specimen shows bone marrow occupation by neoplastic cells (blue stain) with stromal desmoplasia (light pink stain). Panel C shows positive nuclear staining for NKX3.1 (brown), which is consistent with prostate cancer.

\section{months of androgen deprivation therapy. His back and hip pain slowly improved.}

\section{DISCUSSION}

Prostate cancer is the most common cancer in men with one out of every nine men diagnosed in his lifetime. ${ }^{1}$ While most men initially present with localized, curable disease, ${ }^{1} 4 \%$ present with metastatic disease, an incidence that has been increasing since 2004 . $^{2}$ Despite available treatments, metastatic prostate cancer has a poor prognosis, with an average overall survival of approximately 5 years. ${ }^{3}$

Prostate cancer can be challenging to diagnose. Men with prostate cancer are commonly asymptomatic. Rarely, patients may present with hematuria, bony pain caused by metastasis, or obstructive urinary symptoms like hesitancy or incomplete bladder emptying. Our patient presented with jaw pain, which was ultimately attributed to osteoblastic lesions of the skull. Additionally, his history of urinary frequency and incontinence may have been clues to his underlying diagnosis of prostate cancer.

Prostate cancer screening remains highly nuanced and relies on shared decision-making between patients and healthcare providers. Clinical practice guidelines for early detection of prostate cancer recommend individualized PSA-based serologic screening. ${ }^{4,5}$ Specifically, the United States Preventive Services Task Force recommends screening men aged 55 to 69 years who desire screening and understand the potential harms associated with a positive test result. These harms may include psychological distress and complications from prostate biopsy (eg, pain or infection) or prostate cancer treatment (eg, erectile, urinary, and/or bowel dysfunction). ${ }^{4-6}$ The decision to screen can be guided by individuals' risk factors including African American race, family history, and older age.

While our patient elected not to undergo routine prostate cancer screening, a PSA level was obtained during his diagnostic evaluation and highlights the limitations of PSA-based screening. A PSA level $\leq 4.0 \mathrm{ng} / \mathrm{mL}$ has $21 \%$ sensitivity and $91 \%$ specificity for detecting prostate cancer. ${ }^{7}$ PSA levels above 4.0 $\mathrm{ng} / \mathrm{mL}$ warrant repeat testing and, if persistently elevated, referral to urology for possible prostate biopsy. PSA levels often correlate with burden of disease, and patients with PSA levels $>20 \mathrm{ng} / \mathrm{mL}$ are referred for CT imaging to evaluate for metastat- ic disease. ${ }^{8}$ PSA's poor sensitivity was underscored in a study by Thompson et al who evaluated the incidence of prostate cancer in men participating in the Prostate Cancer Prevention Trial with PSA levels of $<4 \mathrm{ng} / \mathrm{mL}$. ${ }^{9}$ In this study, $15 \%$ of men diagnosed with prostate cancer never had a PSA level $>4 \mathrm{ng} / \mathrm{mL}$. ${ }^{9}$ While most of the cancers in this study were low grade and may have been clinically insignificant, 15\% demonstrated histologic signs of at least intermediate-risk disease. Our patient's PSA level of $2.2 \mathrm{ng} / \mathrm{mL}$ was below the threshold that triggers additional evaluation even though he had widely metastatic prostate cancer.

Our patient's severe jaw and temple pain, weight loss, and progressive hip pain were concerning for GCA. This vasculitis of large- and medium-sized arteries predominantly affects older adults with greatest incidence among those 70 years of age and older. ${ }^{10}$ Symptoms occur because of cranial artery inflammation and may include headache, visual disturbance, erythema or tenderness of the temporal artery, and jaw claudication. Extracranial inflammation may affect the thoracic aorta and its branches and rarely the abdominal aorta and lower limb arteries. Pelvic girdle pain more typically results from associated PMR. Patients may also note systemic symptoms such as fever, weight loss, and fatigue.

Prompt diagnostic testing is important when considering GCA. Most patients with GCA have ESR levels greater than 40 $\mathrm{mm} / \mathrm{hr} .{ }^{11} \mathrm{ESR}$ is a laboratory test that measures the vertical distance erythrocytes travel in a column of blood over 1 hour; in the setting of inflammation, cells form clumps and travel more quickly than individual cells, resulting in a higher value. While moderate elevations in ESR may occur without an identifiable cause, extreme ESR levels_those above $100 \mathrm{~mm} / \mathrm{hr}$, as observed in our patient-are highly suggestive of certain serious conditions, including infection, malignancy, and autoimmune disease such as GCA. ${ }^{12,13}$ Temporal artery biopsy is the gold standard test to diagnose GCA. However, because of noncontiguous inflammation of the temporal artery, biopsies may be falsely negative. Thus, sampling of the contralateral temporal artery may be warranted if suspicion remains high.

As was the case for our patient, PET/CT is not reliable for diagnosing prostate cancer. In contrast to other malignancies (eg, lymphoma, lung cancer), prostate cancer typically does not display increased glucose metabolism. Moreover, the close proximity of the bladder and prostate can interfere 
with imaging interpretation because the fluorodeoxyglucose (FDG) tracer is excreted in the urine. ${ }^{14}$ The reported sensitivity of PET/CT for the diagnosis of prostate cancer ranges from $17 \%-65 \%{ }^{15,16}$ In a small study of men with metastatic prostate cancer, only $18 \%$ of bony metastases were FDG avid, and there was no correlation between FDG avidity and PSA level. ${ }^{15}$ Notably, although PET/CT includes CT imaging, this CT is used to map anatomic landmarks and is not separately interpreted by the radiologist. Thus, even if evidence of prostate cancer was apparent on traditional CT, it may be overlooked on PET/CT.

Several important points regarding diagnostic testing are raised by this case. First, PSA-based screening for prostate cancer may be falsely negative, even in the setting of widely metastatic disease. Second, extreme ESR elevation is a marker for serious underlying disease and warrants a thorough diagnostic evaluation. Finally, PET/CT has limited diagnostic utility in evaluating metastatic prostate cancer because of the normal rates of glucose metabolism. Our patient initially presented with jaw pain, yet his progressive physical symptoms and laboratory abnormalities prompted an evaluation that ultimately

\section{References}

1. Prostate Cancer - Cancer Stat Facts. SEER. Accessed October 23, 2018 https://seer.cancer.gov/statfacts/html/prost.html

2. Li J, Siegel DA, King JB. Stage-specific incidence rates and trends of prostate cancer by age, race, and ethnicity, United States, 2004-2014. Ann Epidemiol. 2018;28(5):328-330. https://doi.org/10.1016/j.annepidem.2018.03.001

3. Sweeney CJ, Chen YH, Carducci M, et al. Chemohormonal therapy in metastatic hormone-sensitive prostate cancer. N Engl J Med. 2015;373(8):737-746 https://doi.org/10.1056/NEJMoa1503747

4. US Preventive Services Task Force. Final Recommendation Statement: Prostate Cancer: Screening. Accessed August 8, 2018. https://www.uspreventiveservicestaskforce.org/Page/Document/RecommendationStatementFinal/ prostate-cancer-screening1

5. American Urological Association. Accessed August 8, 2018. http://www.auanet.org/guidelines/prostate-cancer-early-detection

6. American Cancer Society. American Cancer Society Recommendations for Prostate Cancer Early Detection. Accessed August 8, 2018. https://www. cancer.org/cancer/prostate-cancer/early-detection/acs-recommendations. html

7. Wolf AM, Wender RC, Etzioni RB, et al. American Cancer Society guideline for the early detection of prostate cancer: update 2010. CA Cancer J Clin. 2010;60(2):70-98. https://doi.org/10.3322/caac.20066

8. Mohler JL, Lee RJ, Antonarakis ES, Higano CS, Richey S. NCCN Guidelines Index Table of Contents. Prostate Cancer. 2018:151.

9. Thompson IM, Pauler DK, Goodman PJ, et al. Prevalence of prostate cancer revealed the jaw-dropping diagnosis of PSA-negative, metastatic prostate cancer.

\section{KEY TEACHING POINTS}

- ESR levels greater than $100 \mathrm{~mm} / \mathrm{hr}$ are highly suggestive of certain serious conditions including infection, autoimmune disease, and malignancy.

- PSA-based screening for prostate cancer can result in falsenegative test results. In one study, 15\% of men diagnosed with prostate cancer never had a PSA level greater than 4 $\mathrm{ng} / \mathrm{mL}$ (ie, the level at which repeat laboratory testing and/or referral to urology for possible prostate biopsy is advisable).

- PET/CT has limited diagnostic utility in evaluating metastatic prostate cancer, because prostate cancer cells typically demonstrate normal glucose metabolism.

Disclosures: Drs Griauzde, Northway, Yentz, and Houchens have nothing to disclose. Dr Saint reports personal fees from ISMIE Mutual Insurance Company during the conduct of the study, as well as personal fees from Jvion and Doximity outside the submitted work.

among men with a prostate-specific antigen level $\leq 4.0 \mathrm{ng}$ per milliliter. $N$ Engl J Med. 2004:350(22):2239-2246. https://doi.org/10.1056/NEJMoa031918

10. Pioro MH. Primary care vasculitis: Polymyal gia rheumatica and giant cell arteritis. Prim Care. 2018;45(2):305-323. https://doi.org/10.1016/j.pop.2018.02.007

11. Salvarani C, Hunder GG. Giant cell arteritis with low erythrocyte sedimentation rate: frequency of occurrence in a population-based study. Arthritis Rheum. 2001;45(2):140-145. https://doi.org/10.1002/1529-0131(200104)45:2< 140::AID-ANR166>3.0.CO;2-2

12. Brigden ML. Clinical utility of the erythrocyte sedimentation rate. Am Fam Physician. 1999:60(5):1443-1450.

13. Daniels LM, Tosh PK, Fiala JA, Schleck CD, Mandrekar JN, Beckman TJ. Extremely elevated erythrocyte sedimentation rates: Associations with patients' diagnoses, demographic dharacteristics, and comorbidities. Mayo Clin Proc. 2017;92(11):1636-1643. https://doi.org/10.1016/j.mayocp.2017.07.018

14. Powles T, Murray I, Brock C, Oliver T, Avril N. Molecular positron emission tomography and PET/CT imaging in urological malignancies. Eur Urol. 2007;51(6):1511-1521. http://doi.org/10.1016/j.eururo.2007.01.061

15. Yeh SDJ, Imbriaco M, Larson SM, et al. Detection of bony metastases of androgen-independent prostate cancer by PET-FDG. Nucl Med Biol. 1996;23(6):693-697. https://doi.org/10.1016/0969-8051(96)00044-3

16. Perera M, Papa N, Christidis D, et al. Sensitivity, specificity, and predictors of positive ${ }^{68}$ ga-prostate-specific membrane antigen positron emission tomography in advanced prostate cancer: a systematic review and meta-analysis. Eur Urol. 2016;70(6):926-937. https://doi.org/10.1016/j.eururo.2016.06.021 